\title{
Low-pressure Nitriding according to the FineLPN technology in Multi-Purpose Vacuum Furnaces
}

\author{
Kula Piotr $^{1, a}$, Pietrasik Robert ${ }^{1, b}$, Wołowiec Emilia ${ }^{1, c}$, Januszewicz Bartłomiej ${ }^{1, d}$, \\ Rzepkowski Adam ${ }^{1, \mathrm{e}}$
}

${ }^{1}$ Institute of Materials Science and Engineering, Technical University of Lodz, Stefanowski street 1/15, 90-924 Lodz, Poland

apiotr.kula@p.lodz.pl, ${ }^{b}$ robert.pietrasik@p.lodz.pl, 'emilia.wolowiec@p.lodz.pl, dbartlomiej.januszewicz@p.lodz.pl, ${ }^{\mathrm{e} a d a m . r z e p k o w s k i @ p . l o d z . p l ~}$

Keywords: Low-pressure nitriding; vacuum nitriding; nitrides; heat treatment; tool steels; neural networks

\begin{abstract}
Developing steady state models to conduct and control repeatable processes of low-pressure nitriding is not possible in practice as the nitrogen content in a nitrided layer depends not only on the nitriding parameters, but also on the content of alloying elements in steel and the nucleation stage which is difficult to control. Therefore, a new concept of conducting such processes has been proposed. It has been shown that application of an appropriate method of activation of steel parts surface makes the nucleation stage uniform and reduces its duration. A system based on artificial intelligence methods has also been proposed, which enables modelling and control of non-equilibrium processes of low-pressure nitriding of tool steels. This model is based on the "boost-diffusion" schedule of the process.
\end{abstract}

\section{Introduction}

Moulds and dies are important parts of details subjected to thermal and thermo-chemical treatment in service and corporate hardening plants. Used in pressure die casting and forging, the elements are usually subjected to intensive mechanical and thermal loads at high temperatures. High durability of such tools is ensured partly by using the appropriate materials (high-alloy tool steels), but the optimum properties of dies can be achieved by appropriate thermal or thermo-chemical processing. One of the methods of improving durability of such tools is to combine volumetric thermal processing with precision nitriding, which is nowadays carried out in separate devices. However, it is possible to conduct a process of low-pressure nitriding of tool steels in a multi-purpose vacuum furnace, provided the physical model of a multi-segment process is developed and supported with an experimental learning base. Accumulation of credible learning base has been regarded as a pre-condition for the success of the project, because the processing parameters and each additional element introduced in the material are very important for the ultimate material properties [1-5]. All this was an immediate reason for taking up the study aimed at developing a physical model of multi-segment low-pressure nitriding in a "boost-diffusion" process.

\section{Low-pressure nitriding by the FineLPN (Fine Low Pressure Nitriding) method}

In its broad sense, nitriding is a thermo-chemical process which consists in saturation of the surface layer of steel parts of machines and equipment with nitrogen in order to achieve a very hard and abrasion-resistant surface, while maintaining the desired properties and structure of the core. The designed material properties after the nitriding process is completed must remain closely associated with the method of its ultimate use. As a consequence, the sample temperature and the properties and structure of the top layer of the part being processed must be selected on a case-by-case basis, which requires considerable experience or support. Moreover, unlike in low-pressure carburising, growth of as many as three layers must be controlled in this case: $\varepsilon, \gamma$ ' and the diffusive layer. However, the nitrided layers are widely used in contemporary machine technology, mainly in surface hardening of high-load abrasive connection elements. 
Nowadays, nitriding is usually carried out on an industrial scale in atmospheric or ionic furnaces; however, as has been mentioned earlier, a nitriding process can be conducted in multi-purpose vacuum furnaces, especially when relatively small case depth is required. It is an important advantage of low-pressure nitriding that the number of processing devices is reduced because the entire thermal process is conducted within one furnace operation cycle, through the following stages: quenching, repeated tempering and final nitriding $[6,7,8]$.

Due to low pressures of the nitriding atmosphere inside an HPGQ furnace during the low-pressure nitriding process, it is mainly applied in processing of moulds, dies and other parts made of tool steel. Relatively short, several-hour long processes of nitriding such steels result in formation of very hard and thin layer, which improves the utility parameters of such tools. Moreover, vacuum-nitrided layers are less brittle than those formed by gas-nitriding, both within the limited zone of nitrides and in the zone of external nitriding.

Low-pressure thermo-chemical steel processing is a non-equilibrium process. Therefore, repeatable formation of hardened layers with a set carbon or nitrogen profile and a set hardness distribution in a wide range of engineering materials must be based on a reliable computer simulation of the process in all its phases. Therefore, creation of an experimental learning base has been planned in the form of a set of profiles of structure and hardness for four selected types of alloy tool steel, nitrided in a multi-segment process according to a schedule which included different numbers and duration of "boost - diffusion" segments.

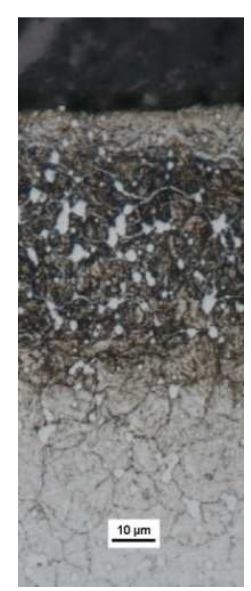

1

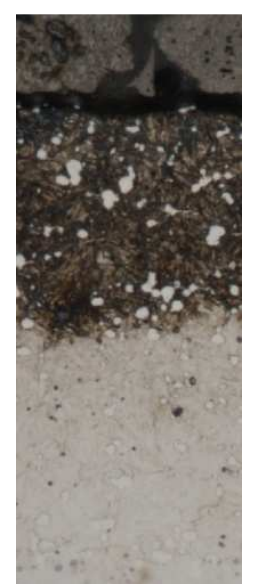

2

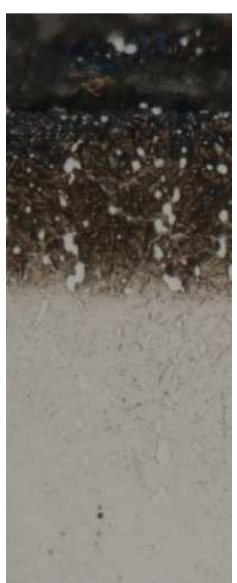

3

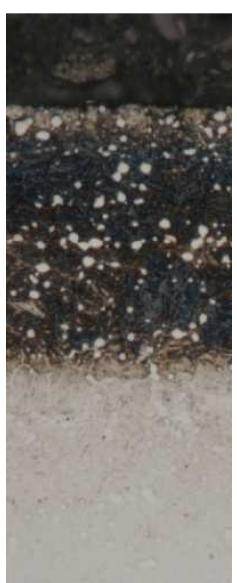

4

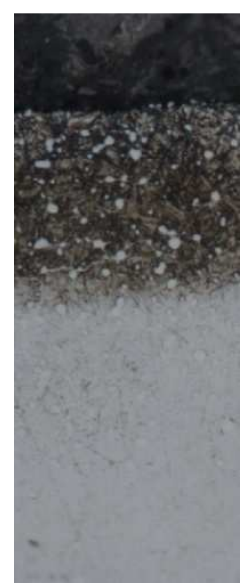

5

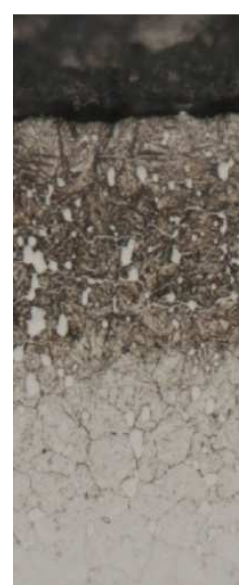

6

Fig. 1. The effect of the activation method on the nitrided layer morphology - process: nitriding in the FineLPN technology, $540^{\circ} \mathrm{C}$, duration: $3 \mathrm{~h}$, material: HS6-5-2 (1.3343).

The results achieved in the qualitative and quantitative studies of samples made of tool steels were used to develop a multi-layer feedforward artificial neural network, whose task is to represent the relationships between the input parameters of low-pressure nitriding and the technological properties of the top layer of the detail being processed, especially the hardness profile and characteristics of the layer thus formed: its thickness, properties and structure.

\section{Experiments}

Selection of the study material and the experiment plan was based on the capabilities of a multi-purpose vacuum furnace and the chemical composition of the steel and on how commonly it is used in industry: HS6-5-2 (1.3343), X37CrMoV51 (1.2343), 55NiCrMoV7 (1.2714) and X165CrMoV12 (1.2379). Subsequently, each material was used for making samples with the diameter of $\phi 28 \mathrm{~mm}$ and thickness of $10 \mathrm{~mm}$, which were quenched, tempered at $570^{\circ} \mathrm{C}$ and ground on both sides. 
Nitriding processes include a stage of incubation and to precipitate the process, it was decided to activate the surface. To this end, six different substances were developed and comparative tests were conducted (Fig. 1); subsequently, the one were chosen which accelerated the case formation while maintaining its optimum morphology.

In the next stage, the appropriate ammonia flow rate was established relative to the charge elements area. A series test processes were conducted and the relationship was determined which allowed to set the optimum flow rate.

Subsequently, a series of processes were conducted at different temperatures $(793 \mathrm{~K}, 813 \mathrm{~K}$ and $833 \mathrm{~K}$ ) with different segmentation. The effect of the parameters on the morphology and properties of the surface layers was examined. Examples of results are shown in Fig. 2 and 3.
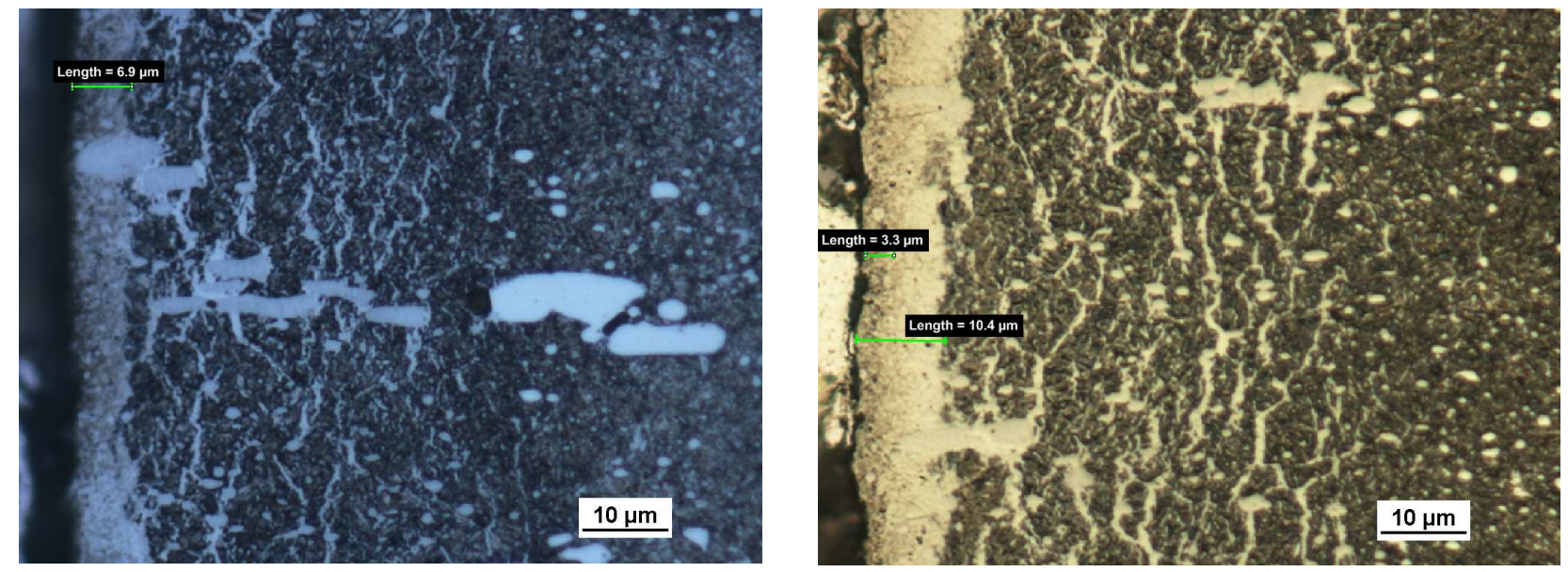

Fig. 2. Morphological structure of X153CrMoV12 after continuous low-pressure nitriding for $6 \mathrm{~h}$ in temp. a) $793 \mathrm{~K}$, b) $813 \mathrm{~K}$. Optical microscopy images, samples were etched by 4\% HNO3/ethanol.
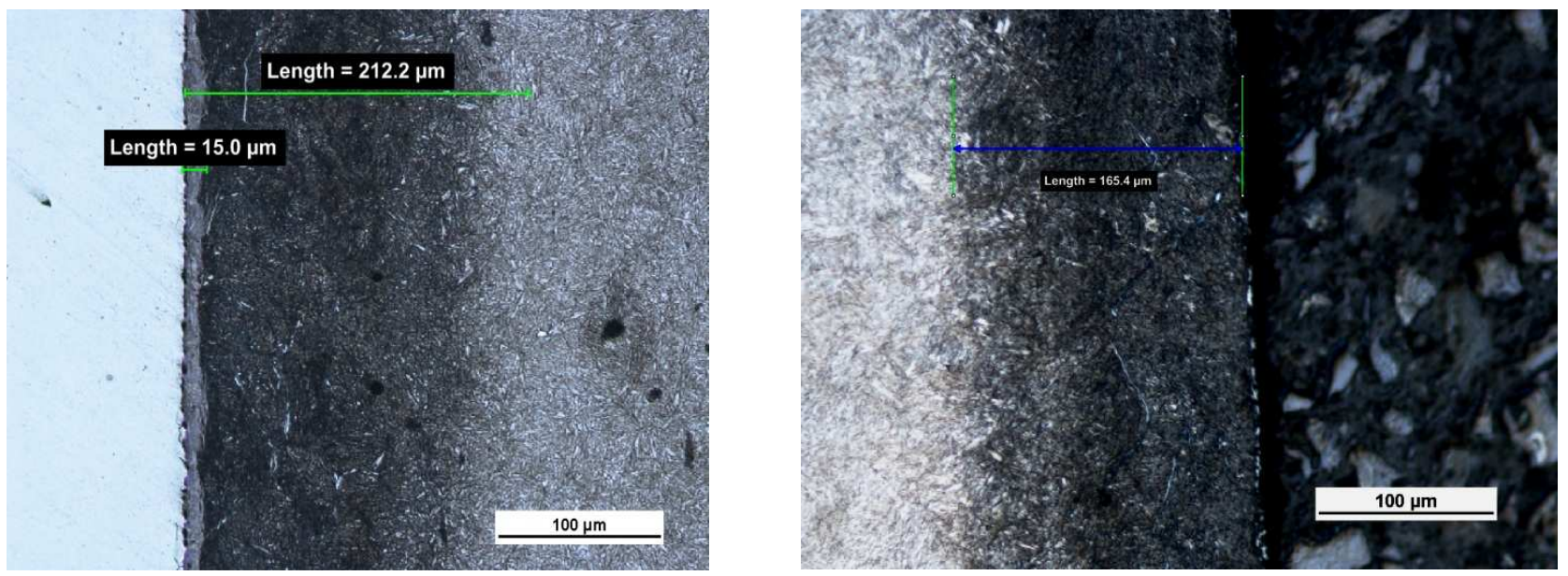

Fig. 3. Morphological structure of X37CrMoV51 steel after low-pressure nitriding in temp. $833 \mathrm{~K} \mathrm{a}$ ) one segment $6 \mathrm{~h}$ process, b) two-segment process with a 4-hour of saturation, and a 2-hour of diffusion. Optical microscopy images, samples were etched by 4\% HNO3/ethanol.

As a result, data were accumulated which were used to develop the SimLPN module.

\section{A computer system supporting $\operatorname{SimLPN}$ (Simulation Low Pressure Nitriding)}

The essence of the experiments lay in accumulation of a vast learning database, concerning the course of low-pressure nitriding processes, needed to examine and describe the kinetics of the processes, in particular to develop a mathematical and neural model of growth of a uniform nitrided case on tool steels, with a desired hardness profile and structure - the optimum in terms of the conditions in which tools are used. 


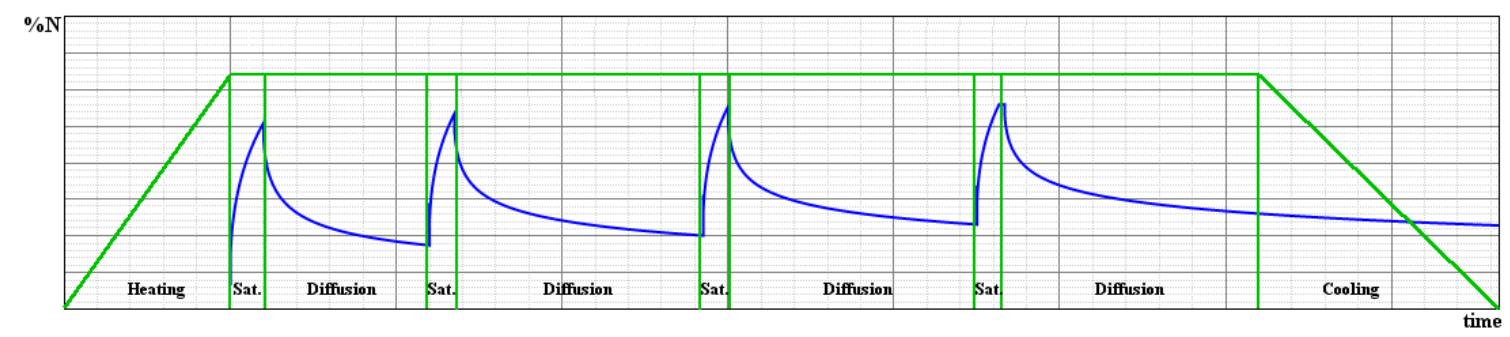

Fig. 4. A "boost-diffusion" process.

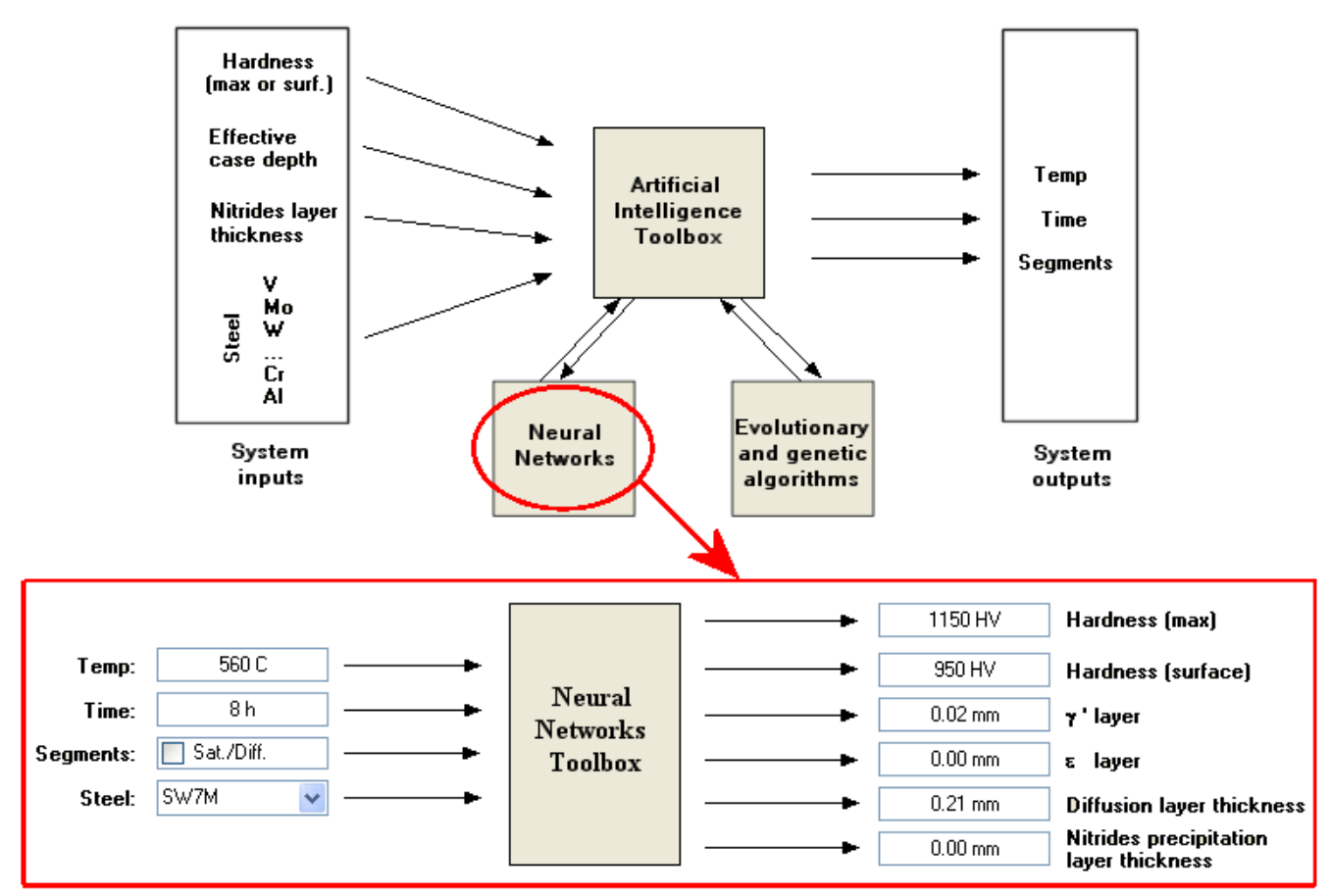

Fig. 5. An example of forecasting the results of nitriding with a SimLPN module.

The project resulted in a consistent SimLPN (Simulation Low Pressure Nitriding) information system, intended for designing, simulating and optimising processes of multi-segment low-pressure nitriding of parts made of tool steels. The system has been developed as a tool which enables implementation of the multi-stage technology of low-pressure nitriding - FineLPN (Fine Low Pressure Nitriding) - as a technological option to be carried out in multi-purpose vacuum furnaces and one which considerably improves the competitive advantage of such devices.

The concept of the programme was based on a modular structure. The computational modules operate independently, exchanging information, whereas the computational core of the system is a module which implements advanced methods of artificial intelligence, especially operation of artificial neural networks. The module implements a physical model and its functional solution for multi-segment low-pressure nitriding in a "boost-diffusion" process (Fig. 4). The diagram of a network operation is shown in Fig. 5.

Experimental verification of the system has been described in successive publications [1, 9-11].

\section{Summary}

Nitriding is a widely applied method of thermo-chemical processing, which enables considerable improvement of utility parameters of tools, thereby extending their life period. Low-pressure nitriding by the FineLPN method is an interesting alternative to be applied in processing elements made of tool steel, such as moulds or dies, reducing the time needed for their processing and the number of devices needed to carry it out. 
The SimLPN information system resulting from the work is an excellent tool which can be used for forecasting structure and, consequently, properties of nitrided case on tool steel depending on the process parameters. It is an example of model operations which start with fundamental research and end with industrial applications.

\section{Acknowledgements}

The study was conducted as part of the project INNOTECH-K1/IN1/5/15/93/96/NCBR/12 and project No. 5216/B/T02/2010/39.

\section{References}

[1] P. Kula, Surface layer engineering, Lodz Technical University, Lodz, 2000.

[2] P. Kula, E. Wolowiec, A. Rzepkowski, B. Januszewicz, M. Wentlandt, Możliwości azotowania stali narzędziowych w uniwersalnym piecu próżniowym [Possibility of nitriding tool steel in a multi-purpose vacuum furnace], Material Engineering 4 (182) (2011) 506-509.

[3] P. Kula, M. Korecki, R. Pietrasik, E. Wolowiec, K. Dybowski, Ł. Kołodziejczyk, R. Atraszkiewicz, M. Krasowski, FineCarb - the flexible system for low pressure carburizing, Journal of The Japan Society for Heat Treatment 49 (2009) 133-136.

[4] M. Kulka, A. Pertek, L. Klimek, The influence of carbon content in the borided Fe-alloys on themicrostructure of iron borides. Materials Characterization 56/3 (2006) 232-240.

[5] A. Pertek, M. Kulka, Two-step treatment carburizing followed by boriding on medium-carbon steel, Surface and Coatings Technology 173 (2003) 309-3141.

[6]. P. Kula, R. Pietrasik, K. Dybowski, M. Krasowski, S. Pawęta, M. Korecki, PreNitLPC zaawansowana technologia wysokotemperaturowego nawęglania próżniowego - efekty i zastosowania [PreNitLPC - an advanced technology of high-temperature low-pressure carburising - effects and applications], Proc. XIIIth Seco/Warwick Seminar Swiebodzin, Poland (2010).

[7] M. Korecki, P. Kula, J. Olejnik, New capabilities in HPGQ vacuum furnaces, Industrial Heating 3/2011, 2011.

[8] M. Korecki, J. Olejnik, M. Bazel, P. Kula, R. Pietrasik, E. Wolowiec, Multi-purpose LPC+LPN+HPGQ 25 bar N2/He single chamber vacuum furnaces, Proc. 3rd International Conference on Heat Treatment and Surface Engineering of Tools and Dies, Wels, Austria (2011), pp. 161-168.

[9] P. Kula, R. Pietrasik, K. Dybowski, R. Atraszkiewicz, E. Wołowiec, M. Korecki, J. Olejnik, New technological pathways for universal vacuum furnaces, Proc. 18th IFHTSE - International Federation for Heat Treatment and Surface Engineering, Rio de Janeiro, Brazil (2010).

[10]P. Kula, M. Korecki, J. Olejnik, R. Pietrasik, E. Wolowiec, Low pressure nitriding - FineLPN the new option for LPC + HPGQ vacuum furnaces, Proc. Furnaces of North America, Orlando, Florida (2010).

[11]M. Korecki, J. Olejnik, P. Kula, R. Pietrasik, E. Wołowiec, Multi-purpose LPC+LPN+HPGQ 25 bar N2/He single chamber vacuum furnaces, Proc. ASM 2011 Heat Treating Society Conference and Exposition, Cincinnati, Ohio (2011), pp. 309-314. 